\title{
Calibration and assessment of electrochemical air quality sensors by co-location with regulatory-grade instruments
}

David H. Hagan et al.

Correspondence to: Jesse Kroll (jhkroll@mit.edu)

The copyright of individual parts of the supplement might differ from the CC BY 4.0 License. 


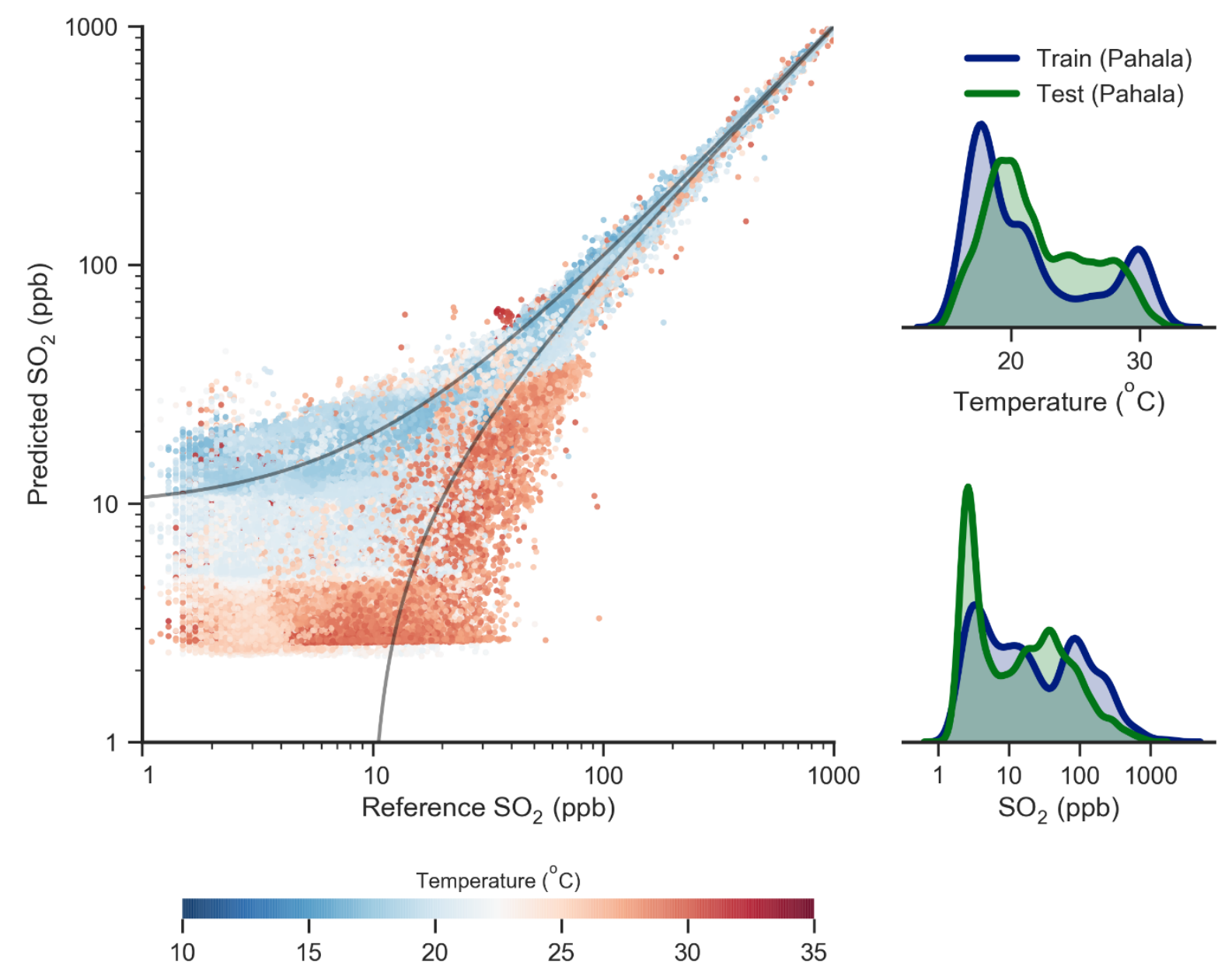

Figure S1: Hybrid regression results for node S02-02 when trained using data from the Pahala AQ station ( 2 days) and validated using data from the following 4 months at the same physical location. This figure is for comparison with Fig 7, which shows results from a second sensor (S-04) which was trained at the Pahala AQ station for the same 2-day period, followed by relocation to the Hilo AQ station over the same period. The correlation coefficient $\left(\mathrm{r}^{2}\right)$ is 0.986 and the RMSE is $9.6 \mathrm{ppb}$. The somewhat better performance of this sensor relative to the one at Hilo (Fig. 7) is due to the better (thought not perfect) match of training-set and test-set conditions (right panels). The difference in this calibration and the calibration of the same sensor over the full training set (Fig. 5, Table 1) is likely due to the reduction in training data, leading to a narrower range of $V_{W E}, V_{A E}, T$ combinations used in the kNN neighbors algorithm. 


\section{Table S1. Performance of all sensors at low(er) concentrations}

To compliment Table 1 and expand on Section 3.6, we have included a complete table of results for the evaluation of sensors given the dataset is comprised only of points where $\mathrm{SO}_{2}$ was lower than $25 \mathrm{ppb}$. Here, $N$ is the total number of 1-minute data-points used in the evaluation.

k Nearest Neighbors

\begin{tabular}{c|c|cc|}
\hline Node no. & $\mathbf{N}$ & $\mathbf{R M S E}$ & $\mathbf{r}^{2}$ \\
SO2-01 & 13,395 & 3.02 & 0.786 \\
SO2-02 & 37,578 & 2.89 & 0.788 \\
SO2-03 & 752 & 1.39 & 0.946 \\
SO2-04 & 1,615 & 2.02 & 0.884 \\
SO2-05 & 1,341 & 2.2 & 0.851 \\
SO2-06 & 18,206 & 2.68 & 0.824 \\
SO2-07 & 1,597 & 1.34 & 0.947 \\
SO2-10 & 1,594 & 2.15 & 0.864 \\
SO2-12 & 587 & 1.83 & 0.912 \\
\hline Median & & 2.15 & 0.864 \\
St. Dev. & & 0.61 & 0.061 \\
\hline
\end{tabular}

